\author{
R. Heinkelmann · J. Böhm • S. Bolotin • \\ G. Engelhardt - R. Haas • R. Lanotte • \\ D. S. MacMillan · M. Negusini - E. Skurikhina • \\ O. Titov · H. Schuh
}

\title{
VLBI-derived troposphere parameters during CONT08
}

Received: date / Accepted: date

R. Heinkelmann (corresponding author)

Deutsches Geodätisches Forschungsinstitut (DGFI)

Alfons-Goppel-Str. 11, 80539 Munich, Germany

E-mail: heinkelmann@dgfi.badw.de

J. Böhm · H. Schuh

Institute of Geodesy and Geophysics (IGG), Vienna University of Technology

Gusshausstr. 27-29, 1040 Vienna, Austria

E-mail: johannes.boehm@tuwien.ac.at

S. Bolotin and D. S. MacMillan

NVI Inc. and NASA/Goddard Space Flight Center (GSFC)

Code 698, Greenbelt, Md 20771, USA

E-mail: dsm@gemini.gsfc.nasa.gov

G. Engelhardt

Bundesamt für Kartographie und Geodäsie (BKG)

Karl-Rothe-Str. 10-14, 04105 Leipzig, Germany

E-mail: gerald.engelhardt@bkg.bund.de

R. Haas

Department of Earth and Space Science, Chalmers University of Technology

Onsala Space Observatory (OSO), 43992 Onsala, Sweden

E-mail: rudiger.haas@chalmers.se

R. Lanotte

Centro di Geodesia Spaziale (CGS)

PO Box 11, 75100 Matera, Italy

E-mail: roberto.lanotte@e-geos.it

M. Negusini

Istituto di Radioastronomia, Istituto Nazionale di Astrofisica (INA)

Via P. Gobetti 101, 40129 Bologna, Italy

E-mail: negusini@ira.inaf.it

E. Skurikhina

Institute of Applied Astronomy (IAA)

nab. Kutuzova, 10, St. Petersburg 191187, Russia

E-mail: sea@ipa.nw.ru

O. Titov

Geoscience Australia (AUS)

PO Box 378, Canberra, 2601, ACT, Australia

E-mail: oleg.titov@ga.gov.au 


\begin{abstract}
Time-series of zenith wet and total troposphere delays as well as north and east gradients are compared, and zenith total delays $(Z T D)$ are combined on the level of parameter estimates. Input data sets are provided by ten Analysis Centers (ACs) of the International VLBI Service for Geodesy and Astrometry (IVS) for the CONT08 campaign (2008 August 12 - 26). The inconsistent usage of meteorological data and models, such as mapping functions, causes systematics among the ACs, and differing parameterizations and constraints add noise to the troposphere parameter estimates. The empirical standard deviation of ZTD among the ACs with regard to an unweighted mean is $4.6 \mathrm{~mm}$. The ratio of the analysis noise to the observation noise assessed by the operator/software impact (OSI) model is about 2.5. These and other effects have to be accounted for in order to improve the intratechnique combination of VLBI-derived troposphere parameters. While the largest systematics caused by inconsistent usage of meteorological data can be avoided and the application of different mapping functions can be considered by applying empirical corrections, the noise has to be modeled in the stochastic model of intra-technique combination. The application of different stochastic models shows no significant effects on the combined parameters but results in different mean formal errors: the mean formal errors of the combined ZTD are $2.3 \mathrm{~mm}$ (unweighted), $4.4 \mathrm{~mm}$ (diagonal), $8.6 \mathrm{~mm}$ (variance component estimation), and $8.6 \mathrm{~mm}$ (operator/software impact, OSI). On the one hand, the OSI model, i.e. the inclusion of off-diagonal elements in the cofactor-matrix, considers the reapplication of observations yielding a factor of about two for mean formal errors compared to the diagonal approach. On the other hand, the combination based on variance component estimation shows large differences among the variance components and exhibits a comparable scaling of formal errors. Thus for the combination of troposphere parameters a combination of the two extensions of the stochastic model is recommended.
\end{abstract}

Keywords VLBI · troposphere parameters · intra-technique combination

\title{
1 Introduction
}

The continuous Very Long Baseline Interferometry (VLBI) campaign 2008 (CONT08 ${ }^{1}$ ) was a fifteen day period of continuous geodetic VLBI observations accompanied by other techniques motivated by the International VLBI Service for Geodesy and Astrometry (IVS). Eleven stations of the IVS network took part during August 12 to August 26, 2008. Like previous CONT campaigns, such as CONT05, CONT02, CONT96, this technically very demanding campaign allows a variety of specific scientific investigations including detailed analyses of the troposphere parameters.

The four types of troposphere parameters defined at a specific station are (in $\mathrm{mm}$ ):

1 http://ivscc.gsfc.nasa.gov/program/cont08/ 
- zenith hydrostatic delay $(Z H D)$ or zenith total delay $(Z T D=Z H D+Z W D)$,

- zenith wet delay $(Z W D)$,

- north-south gradient $\left(G_{N}\right)$, and

- east-west gradient $\left(G_{E}\right)$.

Actually these parameters account for the total non-dispersive effects of the entire atmosphere on the measurement signal and thus should be named non-dispersive or neutrosphere (neutral atmosphere) parameters. However, due to the increasing gas density the troposphere contributes the largest effects and in particular contains almost all of the humidity, i.e. water vapor and clouds. Thus, the incorrect term troposphere parameter is justified and will be kept here for the sake of continuity.

The well-known neutral atmosphere delay model of space-geodetic techniques at radio wavelengths, such as VLBI, GNSS (Global Navigation Satellite Systems), and DORIS (Doppler Orbitography and Radiopositioning Integrated by Satellite)

$$
\begin{aligned}
L(\epsilon, a) & =m f_{h}(\epsilon) \cdot Z H D+m f_{w}(\epsilon) \cdot Z W D+ \\
& +m f_{g}(\epsilon) \cdot\left[G_{N} \cdot \cos (\alpha)+G_{E} \cdot \sin (\alpha)\right]
\end{aligned}
$$

relates the troposphere parameters to the observed delay in line-of-sight $L$ depending on the elevation $\epsilon$ and azimuth angles $\alpha$ and the known mapping functions: the hydrostatic mapping function $m f_{h}$, the wet mapping function $m f_{w}$, and the gradient mapping function $m f_{g}$. The hydrostatic part $Z H D$ contributes about $90 \%$ of the delay in the zenith direction. $Z H D(\mathrm{~mm})$ can be very precisely computed using the surface air pressure $p(\mathrm{hPa})$

$Z H D=\frac{2.2768 \pm 0.0005 \cdot p}{1-0.00266 \cdot \cos (2 \varphi)-0.00028 \cdot h}$

where $\varphi$ denotes the latitude and $h$ the height above the geoid $(\mathrm{km})$ of the phase center of the geodetic instrument (Davis et al. 1985). The latitude and height do not have to be known precisely for the evaluation of the above equation. Thus, the surface air pressure contributes the dominant factor for the uncertainty of $Z H D$. There is no appropriate deterministic model to compute the remaining non-hydrostatic part, mostly caused by the dipole-moment of water vapor and thus referred to as the wet part $(Z W D)$. Therefore, parameters of a wet troposphere model are estimated along with the geodetic parameters.

It has been shown that $Z W D$ s obtained by space-geodetic techniques can improve meteorological models and can provide an independent validation method for climate time-series (Elgered 2001; Heinkelmann 2008). For geodesy troposphere parameters are at least indirectly relevant, since troposphere parameters, geodetic parameters, such as the station positions, and other groups of parameters, can be significantly correlated (cf Nothnagel et al. 2002). In contrast to the station coordinates, which are 
usually defined constant over the duration of the VLBI-session, the troposphere parameters are defined with a much higher temporal resolution. Hence, the troposphere parameters reflect sub-diurnal effects and have the potential to absorb short-period fluctuations and systematics of other parameters, such as the station coordinates, as well. Due to these characteristics the troposphere parameters contribute important information about the consistency of the entire solution. Recognizing this, the IVS has monitored troposphere parameters of the ongoing rapid-turnaround type of session (IVS-R1, IVS-R4) since its start in 2002 (Böhm et al. 2002). IVS combined series of troposphere parameters are determined and provided to the user community. The IVS troposphere products are a long-term combination ${ }^{2}$ with the focus on climate applications (Heinkelmann et al. 2007) and a rapid combination ${ }^{3}$ based on models and analysis options defined by IERS Standards. Since 2002 the combination model of the IVS rapid troposphere product (Schuh and Böhm 2003) has not been revised, and the deviations among the contributing ACs were found to slightly increase, which could be caused by increasingly different models applied by the analysis centers (Heinkelmann 2009).

Within the scope of this article we investigate possible improvements of the combination model of troposphere parameters: on the one hand, systematics caused by different analysis options are accounted for by applying empirical corrections; on the other hand, analysis and model noise introduced by the ACs are considered by an extended stochastic model of intra-technique combination. In the next section the troposphere data provided by the individual ACs are presented, and pre-processing steps are described. Thereafter the individual AC solutions are compared with each other and with a mean series in order to empirically assess the quality of the reported troposphere parameter time-series and to reject outliers. Then, various model impacts are highlighted, and corresponding corrections are derived. The empirical measure of the quality of the reported series is thereafter compared with the theoretical formal errors obtained by various extended combination models, which are introduced in the following section. In a last section we conclude our investigations with recommendations and finish with an outlook on possible further extensions of the intra-technique troposphere combination model.

\section{Input data and pre-processing}

The input data are time-series of the troposphere parameters $\left(Z T D, Z W D, G_{N}\right.$, and $\left.G_{E}\right)$ and formal errors of the troposphere parameter estimates $\left(\sigma_{Z W D}, \sigma_{G N}, \sigma_{G E}\right)$ provided by ten IVS ACs (tab. 1). Depending on the software and the operator, the following models and parameterizations are applied for the estimation of zenith delays: continuous piece-wise linear function (PWLF) using least-squares estimation in the Gauss-Markov-model (cf Koch 1997); a random walk stochastic process (RW) ap-

2 http://www.dgfi.badw.de/?196

3 http://www.dgfi.badw.de/?194 
plying the Kalman-filter (cf Gelb 1974); the Square Root Information Filter (Bierman 1977); or the least-squares collocation technique (Titov and Schuh 2000). The gradients are estimated with similar models. The temporal resolution of the zenith delays is conventionally fixed to one hour at integer UT hours. The gradient epochs are also defined at integer UT hours, but the temporal resolution and parameterization are left to the analyst. While in the Gauss-Markov-model the parameters can be directly defined at the conventional epochs with one hour duration, the filter and least-squares collocation techniques provide estimates at the observation epochs. The corresponding ACs then report mean values of the estimates within intervals of one hour duration around the conventional epochs. The main difference between the approaches is that the observations contributing to one parameter in the case of the Gauss-Markov-model are simultaneously adjusted introducing correlations between the observations, while if applying one of the other techniques the computation depends only on the specific observation, and no correlations appear between observations.

The following pre-processing of the input data is done:

(i) Apart from the other ACs, AUS provides the original output of the RW process: one troposphere parameter estimate per observation and no formal errors. The estimates are averaged to the conventional 1-hourly bins centered at integer UT hours. For the formal errors empirical values are introduced, determined by the standard deviation of the estimates within the hourly intervals.

(ii) IGG delivers troposphere parameters with a 30 min resolution. Both zenith delays $(Z W D$ s and $Z T D \mathrm{~s})$ are averaged to the conventional 1-hourly interval by a simple approach:

$Z D_{t}=1 / 4 \cdot\left(Z D_{t-0.5 h}+2 \cdot Z D_{t}+Z D_{t+0.5 h}\right)$ with $t=0 U T, 1 U T, \cdots, 23 U T$ of each day and small adaptations at the beginning and at the end of the CONT08 time span. The same procedure is applied to the corresponding formal errors for the IGG data.

(iii) Some of the ACs that apply the Gauss-Markov-model (BKG, DGFI, GSFC, IGG, INA, and OSO) also report troposphere parameters at epochs without observations. Parameters that are determined purely by the constraints are considered as unreliable and are eliminated.

(iv) In the case of IAA and MAO some of the troposphere parameters in the beginning of a session are occasionally missing or show significantly larger formal errors compared to the average level of formal error of the AC. IAA performs outlier detection and, hence, does not forward certain estimates. Besides the conventional analysis on a session-by-session basis, IAA also provides a solution where the CONT08 sessions are analyzed all together. In this solution outliers and significantly larger formal errors are found only in the beginning of the entire CONT08 period, i.e. in the beginning of the first session. The effects are caused by the application of the filter process in which the initial values differ significantly from the real troposphere conditions and the filter is applied only in forward mode. This effect can be avoided if a backward filter is applied after the forward filter. For our investigations 
the conventional (session-wise) solution of IAA is used, and unreliable troposphere parameters in the beginning of each session are considered as outliers. Similar but smaller effects are seen in the MAO data.

(v) Another pre-processing step handles overlapping estimates at the transition from one session to the next. For example this is the case for BKG gradients. BKG reports two estimates at the same epoch, one at the end of a session and another at the beginning of the next session. To ensure a unique value for each epoch the overlapping values are averaged.

(vi) At the last pre-processing step any missing values are artificially filled and marked as outliers so that an equally spaced time grid with one-hourly sampling underlies each input series of each parameter and AC. A one-hourly series is also generated for the gradients after lower resolved estimates are interpolated in an appropriate way. These input data are used for the following comparisons.

\section{Comparison of troposphere parameters provided by various IVS Analysis Centers}

In this section the one-hourly sampled input data are compared to each other and to an unweighted epoch-wise mean. The comparison allows identification of systematics caused by varying analysis options and detection of outliers which could otherwise significantly distort the determination of the stochastic model of the combination (sec. 4). Before the outlier detection, systematic effects caused by different analysis options are identified and considered.

3.1 Effects caused by inconsistent analysis options

The term analysis option denotes any relevant decision or choice taken during the course of analysis. An analysis option belongs to one of the four categories:

- meteorological data used for the analysis,

- models,

- parameterizations and constraints, or

- data editing, i.e. elimination or reweighting of observations.

Some of the VLBI analysis options significantly affect the troposphere parameters. Heinkelmann (2008) identified significant effects on the troposphere parameters based on VLBI observations between 1984 and 2008 when applying the models available about mid-2008. It is uncertain whether these effects apply exactly for the CONT08 campaign; therefore, they are used as reference but are revised in this section. Some analysis options which can systematically affect the troposphere estimates were conventionally fixed for the analysis and are therefore common to all ACs: (i) the gradient model is MacMillan (1995), 
(ii) the station coordinates are adjusted together with the troposphere parameters, and (iii) troposphere parameters are defined at integer UT; the zenith delays have an hourly resolution (see sec. 2). The other analysis options were left to the analyst's choice, either because none of the available options is undoubtedly superior, or because a specific option is not available to all ACs. The different analysis options of the ten IVS ACs relevant for the determination of troposphere parameters are summarized in tables $(2,3,4)$. Stochastic (noise) differences are considered in the combination model (sec. 4). This section deals with the determination of empirical corrections for deterministic (systematic) differences caused by inconsistent analysis options. To assess systematic effects on troposphere parameters, we sequentially vary models, parameterizations and constraints, and data handling used for an otherwise identical solution. Other analysis options are expected to have no significant systematic impact on the estimation of troposphere parameters, and therefore are not considered here.

\subsubsection{Meteorological data}

ZHDs show pronounced diurnal and semidiurnal signals at Hartebeesthoek (fig. 1), Kauai, and Tsukuba (not shown here) and generally agree very well among the ACs at most of the sites. The diurnal and semidiurnal signals are due to $S_{1}$ and $S_{2}$ atmospheric surface pressure tides caused by thermal heating of the sun (Jin et al. 2008). However, ZHDs provided by DGFI are systematically smaller at some sites by up to $15.4 \mathrm{~mm}$ (at Svetloe). DGFI uses the readings of the local pressure sensor with the mean pressure level shifted to the corresponding mean pressure level given through the ECMWF (European Centre for Medium-Range Weather Forecasts) ERA40 or operational analysis. The constant offsets are determined by the entire history of pressure records considering all valid local pressure observations of all available VLBI sessions and breaks are introduced in case of significant jumps of the running mean value (Heinkelmann et al. 2005). The smaller mean value of $Z H D$ s in the example of Svetloe is almost totally compensated through the $Z W D$ estimates $(14.9 \mathrm{~mm})$, yielding no significant mean change of ZTDs $(-0.5 \mathrm{~mm})$. The large $Z H D$ offset of $-15.4 \mathrm{~mm}$ follows from a large difference of the mean ECMWF pressure of $-6.7 \mathrm{hPa}$ with regard to the mean of the local surface pressure readings. At most of the stations unexplained breaks of the running mean value of the pressure readings can be found during the course of time. For example at Zelenchukskaya a pressure break of $11.4 \mathrm{hPa}$ occurred in mid-2007. Since no meta data about the break are available, it is impossible to decide which running mean value is the correct one. A break of $11.4 \mathrm{hPa}$ corresponds to a shift of the height of the pressure sensor of about $90 \mathrm{~m}$. A relocation with such a large height difference is very unlikely. Since such breaks have occurred at almost all sites and calibrations of the local pressure sensors are rather rare, DGFI considers the mean pressure realized by the ECMWF weather model to be more globally consistent and more reliable. During CONT08 no meteorological data were 
recorded at Zelenchukskaya. The ACs use different methods to substitute the missing pressure values at Zelenchukskaya (tab. 2), which is why $Z H D$ s at this specific site differ significantly (fig. 2). For Zelenchukskaya GSFC, IAA, and DGFI replace the missing pressure values by values from a numerical weather model, such as ECMWF. The GSFC $Z H D$ values are additionally offset by about $3.6 \mathrm{~mm}$. BKG, CGS, INA, and OSO apply a constant value of about $882 \mathrm{hPa}$ taken from a standard model of the atmosphere (Berg 1948). While IGG applies the empirical meteorological model GPT (Böhm et al. 2007), AUS uses some constant value close to GPT. The MAO $Z H D$ values are close to GPT also but show some variations at higher frequencies. As shown by Heinkelmann et al. (2009) values derived from numerical weather models, such as ECMWF interpolates that are provided by $\mathrm{IGG}^{4}$, are a valid substitute for missing pressure data. Constant values or other models are not recommended for precise VLBI analyses. During CONT08 and for station Zelenchukskaya the difference between GPT and ECMWF pressure is larger than the difference between standard pressure (Berg 1948) and ECMWF. The inconsistent substitution for missing meteorological data by different ACs causes the largest differences on $Z H D$ s. At the other sites, where pressure values of local sensors provided by IVS are consistently used by the ACs, $Z H D$ s agree very well (fig. 1). The top panels of figs. 1 and 2 show the $Z W D$ differences for each point between the specific AC and the unweighted mean of the ACs. The $Z W D$ estimates at Zelenchukskaya clearly reflect the usage of different pressure values. For the "combined" $Z W D$ s this effect is irrelevant, because the "combined" $Z W D$ s are obtained by subtracting the DGFI $Z H D$ s from the combined $Z T D$ s after the $Z T D$ s are combined.

As seen in equ. 2 the accuracy of $Z H D$ s depends directly on the accuracy of the surface air pressure. Compared to $Z T D \mathrm{~s}, Z W D \mathrm{~s}$ depend much more on the accuracy of $Z H D \mathrm{~s}$. This is due to the fact that $Z H D$ s are the a priori values for the estimation of the $Z W D$ residuals. Thus, $Z H D$ s and $Z W D$ s show a large negative correlation of about -0.9 , a value which depends on the difference between the hydrostatic and wet mapping functions (Heinkelmann 2008). Since $Z T D$ s are the sum of $Z W D$ and $Z H D$, the correlation with the $Z H D$ s is significantly less but cannot be neglected. The reported formal errors of $Z W D$ estimates consider neither the precision nor the accuracy of surface pressure; thus, they are not valid uncertainties of the $Z W D$ s. For the accuracy of $Z W D \mathrm{~s}$ and $Z T D \mathrm{~s}$, the accuracy of the pressure must be considered. While the precision of a pressure sensor is specified by the manufacturer, the accuracy of the pressure is not given and not easily derivable. If, for example, the pressure is supplied by a local pressure sensor, the accuracy of the sensor assessed by calibration and the effects of spatial and temporal interpolations were to be considered involving the quality of the models used for the interpolation. However, pressure sensor calibrations at the geodetic VLBI sites are rare. If the pressure at a site is taken from a numerical weather model (NWM), the pressure is obtained by a

\footnotetext{
4 http://ggosatm.hg.tuwien.ac.at/DELAY/
} 
complex intertwining of many met sensors and the dynamics of the NWM, and the error depends on the location of the sensor within the NWM analysis grid as well. Among various NWMs differences are seen as well which can give only a lower bound on the accuracies of the NWMs. Lacking more information about the pressure, we have to assume a certain average accuracy of the pressure; a rather conservative assumption for the pressure accuracy would be $2 \mathrm{hPa}$. Consequently, for the uncertainty of $Z W D \mathrm{~s}$, we use the reported formal errors of $Z W D$ estimates $\left(\sigma_{Z W D}\right)$ but uniformly increase it by $4.6 \mathrm{~mm}$ applying equ. $2\left(\widetilde{\sigma}_{Z W D}=\sqrt{\sigma_{Z W D}^{2}+4.6^{2} \mathrm{~mm}^{2}}\right)$. The corresponding increase of the uncertainty of $Z T D \mathrm{~s}$ is $0.4 \mathrm{~mm}$.

\subsubsection{Models}

\subsubsection{TRF}

Several terrestrial reference frames (TRF) are used by the ACs to generate session-wise apriori values for station coordinates: ITRF2000 (Altamimi et al. 2002), ITRF2005 (Altamimi et al. 2007), and the VLBI contribution to the ITRF2008 called VTRF2008 (Böckmann et al. 2010). The effect on $Z W D$ s of using the various apriori TRF is negligible if the station coordinates are adjusted by session during parameter estimation. Fixing the station positions on session-wise a priori values specified through the respective catalogue could significantly affect the troposphere parameters (fig. 3).

\subsubsection{Mapping functions}

The Analysis Centers of IVS apply the Vienna Mapping Functions 1 (VMF1), Böhm et al. (2006), currently recommended by IERS (2004), or the New Mapping Functions (NMF), Niell (1996). Switching from NMF to VMF1 causes systematic effects (fig. 4). Although using NMF instead of VMF1 for CONT08 systematically affects the zenith delays, the gradient estimates vary around zero. The maximum mean change of $-1.9 \mathrm{~mm}$ and a maximum increase in standard deviation of $1.4 \mathrm{~mm}$, both for ZTDs, can be found using NMF at Tsukuba, Japan. Asuming VMF1 gives the better result, the differences between two identical DGFI solutions, one with NMF and the other with VMF1, are used as empirical corrections for the zenith delays of the ACs using NMF (CGS, INA, MAO, OSO).

\subsubsection{Apriori gradients}

DGFI initially used the total atmosphere gradients based on the GSFC numerical weather model DAO (MacMillan and Ma 1998) as apriori gradients, while the apriori gradients of the other ACs are zero. Effects of a priori gradients on the troposphere parameters are very small during CONT08 (fig. 4), but can reach very large values at some sites before 1990 (Heinkelmann 2008). Instead of correcting the DGFI solution, another DGFI solution with zero apriori gradients was computed and used for the combination. Using different apriori gradients results in different adjusted gradients, which is due to the application of constraints. 


\subsubsection{Parameterizations and constraints}

Apart from the other analysis options the impact of differing parameterizations and constraints of the ACs that apply the Gauss-Markov-model (tab. 3) is assessed in one step because it is not possible to gather the entire effect from sequential variation. This category of analysis options does not cause systematics but contributes significantly to the noise component (fig. 5).

\subsubsection{Data handling}

The cutoff elevation angle applied by the ACs varies between $0^{\circ}$ and $5^{\circ}$. Since there are no observations below $5^{\circ}$ elevation during CONT08, the use of different cutoff elevation angles is irrelevant. Some analysis centers additionally or alternatively apply a downweighting of observations, below $10^{\circ}$ elevation. Figure 6 shows the impact of data handling on the troposphere parameters. The downweighting using $\cos ^{2}\left(90^{\circ}-\epsilon\right)$ exhibits the largest effects followed by the $\cos \left(90^{\circ}-\epsilon\right)$ downweighting. The extended stochastic model applied by DGFI (Tesmer 2004) generally causes smaller differences with regard to the solution without data editing. The gradient estimates are affected more than the $Z W D$ by the handling of low elevation data because the gradient mapping functions show stronger dependence on the elevation angle than the mapping functions of the zenith delays. No systematic effect can be found when varying the data editing. Nevertheless the noise contribution is again found to be significant (fig. 6).

\subsubsection{Remaining differences}

Comparing the mean of the standard deviations of the ACs to the unweighted mean of the ACs for $Z W D$ s at various sites (tab. 5), significant differences can be observed. At Ny-Ålesund (2.5 mm) and Wettzell $(2.8 \mathrm{~mm})$ very small standard deviations occur, while at Zelenchukskaya $(6.9 \mathrm{~mm})$ and Tsukuba $(6.2 \mathrm{~mm})$ the standard deviations are more than twice as large. The large mean of the standard deviation of $Z W D$ s at Zelenchukskaya is caused by the different $Z H D$ s due to inconsistent treatment of pressure values. At Tsukuba the mean value of $Z W D$ s is about twice as large $(290 \mathrm{~mm})$ as at the other ten sites. Thus the relative standard deviation compared to the mean size of the parameter remains the same. However, large standard deviations are not always accompanied by large mean values of $Z W D$ s: at Hartebeesthoek $(70 \mathrm{~mm})$ and Kauai $(90 \mathrm{~mm})$ the mean values are small but the standard deviations of $5.5 \mathrm{~mm}$ and $4.7 \mathrm{~mm}$, respectively, are rather large. Whenever the reported formal errors of $Z W D$ s are large, the reported formal errors of the gradients are large, too. Gradient estimates show larger variations than zenith delays among the ACs relative to the mean size of the parameter, which usually lies between $\pm 2 \mathrm{~mm}$ for the gradients and between $55 \mathrm{~mm}$ and $240 \mathrm{~mm}$ for 
$Z W D$ s (dry and wet atmospheric conditions). The reported formal errors of gradient estimates are sometimes very small. Thus, to prevent numerical problems, $0.01 \mathrm{~mm}$ is added to the gradient formal errors. The comparison of gradient estimates $\left(G_{N}, G_{E}\right)$ shows that a combination of the reported gradients cannot provide meaningful results. Before gradient estimates can be combined, a common parameterization would need to be defined because the interpolation between estimates of various temporal resolutions causes large scatter. Furthermore the reported adjusted gradients depend on the apriori gradients (fig. 4). To avoid this effect, the constraints have to be omitted or the weights of the constraints have to be significantly reduced, and the apriori zero gradients should be replaced by apriori total gradients, such as the DAO total apriori gradients (MacMillan and Ma 1998). ZTDs of MAO show significant offsets at some sites (not shown here), e.g. at Concepción (18.3 mm) and at Onsala $(-9.7 \mathrm{~mm})$, which are not caused by different pressure values since the $Z H D$ s show only very small or no significant offsets. These offsets are probably caused by inconsistent handling of other correlated parameters, such as the clock parameters. Several analysis software packages are used for geodetic VLBI ${ }^{5}$ : CALC/SOLVE of various versions; MODEST; OCCAM appears in various distributions, mainly independently developed; SteelBreeze; and the recently developed VieVS. The IVS is currently comparing the different software packages on a simulated dataset (Plank et al. 2010). The analysis software is not an analysis option in the genuine sense because it is uncommon for ACs to run more than one analysis software for the same technique in parallel. Since all well defined options are considered separately, the differences caused by the application of various software packages are due to a large number of small deviations, such as different numerical realizations of the involved models, partial derivatives, and other computational issues. If a large number of options affects the estimates to about the same small extent, it is very likely that systematics cancel each other, what is mathematically described by the central limit theorem. Accordingly, such an integrated effect is much more likely to cause noise than systematic differences.

\subsection{Outlier treatment}

The outlier treatment is the step before combination through which robustness can be achieved. Since there is no deterministic model describing the temporal behavior of the troposphere parameters during two weeks of time, outliers cannot be identified with regard to a model; they have to be identified in the sense of abnormal behavior of an individual input with regard to the average behavior of the input. At this stage the average behavior must be approximated by an unweighted mean of the input data because no weights have been determined yet, and the determination of meaningful weights should be

\footnotetext{
${ }^{5}$ http://ivscc.gsfc.nasa.gov/analysis/index.html
} 
performed on an outlier-free observational basis. The detection of outliers could be empirically achieved by visual inspection, because the data size is not too large. Nevertheless, since visual inspection is rather subjective, a data snooping algorithm has been applied: the individual estimate of an AC is compared to the mean of the estimates of the other ACs at the same epoch. If the absolute value of the difference of the estimate and the mean value exceeds the $\kappa$-fold standard deviation of the estimates at the specific epoch, the reported estimate of the $\mathrm{AC}$ will be rejected. For the threshold factor, $\kappa$, various values between 2.5 and 5 are tested. Finally a threshold of $3(\kappa=3)$ is adopted because it enables the data snooping algorithm to detect all visually identified outliers and avoids wrong detections. On the basis of this $3-\sigma$-algorithm, the identified outliers are eliminated.

Through the comparison it is also possible to derive an empirical quality measure for the reported troposphere parameters which will be used to validate the theoretical formal errors determined by the various combination processes (sec. 4). The mean of the standard deviations of the individual AC with regard to an unweighted mean of the ACs over all stations represents an empirical measure of the agreement of VLBI-derived troposphere parameters during CONT08. The mean of the standard deviations of the ACs with regard to the unweighted mean of the ACs over all stations is (4.5 mm, $4.6 \mathrm{~mm}, 0.4 \mathrm{~mm}, 0.4 \mathrm{~mm})$ for $\left(Z W D \mathrm{~s}, Z T D \mathrm{~s}, G_{N}, G_{E}\right)$.

\section{Intra-technique combination of troposphere parameters}

Combinations in geodesy belong to one of the following types:

1. combination on the observation equation level,

2. combination on the normal equation level (Angermann et al. 2004), or

3. combination on the level of parameter estimates.

For intra-technique combinations, the combination on the observation level (1.) is meaningless, because the observations are identical. Compared to the parameter level (3.), the normal equation level (2.) is advantageous because this pre-solution step allows the application of a unique datum for the entire combined model in a consistent way and enables the correlations between parameters to work within the entire combined equation system. Since troposphere parameters are not provided at the normal equation level, only the third type, the combination based on parameter estimates, can be considered so far.

The main point of the paper is to present an improved model for intra-technique combination of troposphere parameters. The improved combination model considers systematics among the input data in terms of empirical corrections (sec. 3.1) and the operator/software impact (OSI, Kutterer et al. 2009) through an extended stochastic combination model (sec. 4.1). Due to the characteristics of the 
input data, $Z T D$ s are combined and $Z W D$ s are derived from the combined $Z T D$ s, whereas gradients are compared but not combined.

\subsection{Theoretical concept}

In this section the general mathematical model of intra-technique combination is introduced; the results are compared later (sec. 4.2). The mathematical model can be applied to the combination on the level of any parameter estimates, not necessarily troposphere parameters. All of the following combination models have the functional model in common but differ in terms of the stochastic models. The following stochastic models are considered for the ZTD combination:

1. the unweighted model,

2. the diagonal model,

3. the variance component model, and

4. the OSI model (Kutterer et al. 2009).

The unweighted model (1.) does not consider any stochastic information. It is used here for comparison demonstrating the amount of impact the other stochastic models have on the parameter estimates and on the formal errors. Model (2.) straightforwardly considers the reported formal errors of the ACs. If the reported formal errors are good approximations of the true uncertainties of the parameters, there will be no significant difference compared to the model (3.) with additional variance components (VCs). Apart from the other three aforementioned stochastic models, the OSI model (4.) considers the fact that all ACs analyze the same original observations (reapplication of observations). Furthermore it allows determination of the ratio of the observation noise to the analysis noise, which is initially unknown, but important for a realistic stochastic combination model.

The linear functional model of intra-technique combination in the Gauss-Markov-model reads as:

$\bar{A} x=\left|l_{1} l_{2} \ldots l_{k}\right|^{T}+\bar{v}=\bar{l}+\bar{v}$

where the input, $l_{i}$, are the ZTD parameters of the ACs. Each is considered an observation and is placed into one common $(3600 \times 1)$-dimensional observation vector $\bar{l}$. The bar denotes the common vector or common matrix. The number of observations, $n=3600$, and the number of unknowns, $u=360$, follow from the 24 1-hourly estimates in the 15 consecutive days of CONT08 provided by the $k=10$ ACs. The actual number of parameters per station can vary due to epochs without observations. $x$ denotes the (ux1) vector of parameters and $\bar{v}$ the (nx1) vector of residuals. The (nxu) design-matrix $\bar{A}$ has a very simple shape:

$\bar{A}=\left|I_{u} \cdots I_{u}\right|^{T}$ 
It gathers ten identity matrices of dimension $(\mathrm{uxu}) I_{u}$ together. In case of an outlier the entry in the A-matrix corresponding to the specific AC and observation is zero, i.e. outlying observations are eliminated. Apart from the trivial cofactor matrix realizing an epoch-wise unweighted mean $Q_{\overline{l l}}=I_{n}$

the most simple and straightforward stochastic model is:

$$
Q_{\overline{l l}}=\left|\begin{array}{cccc}
\operatorname{diag}\left(\sigma_{1}^{2}\right) & 0 & \ldots & 0 \\
0 & \operatorname{diag}\left(\sigma_{2}^{2}\right) & \ddots & \vdots \\
\vdots & \ddots & \ddots & 0 \\
0 & \ldots & 0 & \operatorname{diag}\left(\sigma_{k}^{2}\right)
\end{array}\right|
$$

where the operator $\operatorname{diag}(\cdots)$ denotes that the vector in brackets is expanded to a diagonal matrix. The diagonal model considers the given formal errors $\sigma_{i}$ of the parameter estimates of the $i=1, \cdots, k$ ACs. With variance components $\alpha_{i}^{2}$

$$
Q_{\overline{l l}}=\left|\begin{array}{cccc}
\alpha_{1}^{2} \cdot \operatorname{diag}\left(\sigma_{1}^{2}\right) & 0 & \cdots & 0 \\
0 & \alpha_{2}^{2} \cdot \operatorname{diag}\left(\sigma_{2}^{2}\right) & \ddots & \vdots \\
\vdots & \ddots & \ddots & 0 \\
0 & \ldots & 0 & \alpha_{k}^{2} \cdot \operatorname{diag}\left(\sigma_{k}^{2}\right)
\end{array}\right|
$$

the combined stochastic model is able to overcome a possible different scaling of the formal errors provided by the ACs, although with some more calculation effort. The method to estimate global variance components, i.e. variance components independent from their initial values, is described e.g. by Koch (1997). This approach is broadly used for intra-technique as well as inter-technique combinations in geodesy. If fast variance-component estimation methods are applied, such as the Förstner (1979)method or the more recent Monte-Carlo-based method of Kusche (2003), the computational cost stays acceptable for most applications. The variance-component approach is used to generate the combined troposphere parameters provided to the Institute of Geodesy and Geophysics (IGG) for their further comparisons with other techniques (see Teke et al. this issue). Nevertheless, it has disadvantages in the case of intra-technique combination because it does not account for the reapplication of observations. To further refine the stochastic model of intra-technique combination, we follow Kutterer et al. (2009), who explain the operator/software impact (OSI) of an $\mathrm{AC}, \Delta l_{i}$, on the vector of original observations, $l$, which all ACs initially have in common:

$l_{i}=l+\Delta l_{i}$

The first and second moments ( $E$ : expectation value, $D$ : dispersion) are given by:

$E\left(\Delta l_{i}\right)=0, D\left(\Delta l_{i}\right)=\sigma_{0}^{2} Q_{\Delta l_{i} \Delta l_{i}}$ 
With this approach the OSI is modeled as noise, i.e. it is assumed that no systematics are introduced by the AC. Since all significant systematics are considered through empirical corrections in advance (sec. 3.1), it can be considered that no systematics are present among the AC's solutions anymore. Thus, it appears admissible to assume the aforementioned characteristics (equ. 9) for $\Delta l_{i}$.

A common OSI-parameter $\bar{\alpha}^{2}$ defines the OSI of each AC to be of the same size. It can be obtained through

$\bar{\alpha}^{2}=\frac{k}{\sum_{i=1}^{k} \frac{1}{\alpha_{i}^{2}}}$

where the unknown individual OSI-parameters $\alpha_{i}^{2}$ are given through

$\left|\begin{array}{c}\alpha_{1}^{2} \\ \alpha_{2}^{2} \\ \vdots \\ \alpha_{k}^{2}\end{array}\right|=\frac{1}{\widehat{\sigma}_{l}^{2}} \cdot\left|\begin{array}{c}\widehat{\sigma}_{\Delta l_{1}}^{2} \\ \widehat{\sigma}_{\Delta l_{2}}^{2} \\ \vdots \\ \widehat{\sigma}_{\Delta l_{k}}^{2}\end{array}\right|$

and the variances, $\widehat{\sigma}_{\Delta l_{i}}^{2}$, and covariances, $\widehat{\sigma}_{\Delta l_{i} \Delta l_{j}},(i<j)$, are determined through the equation system

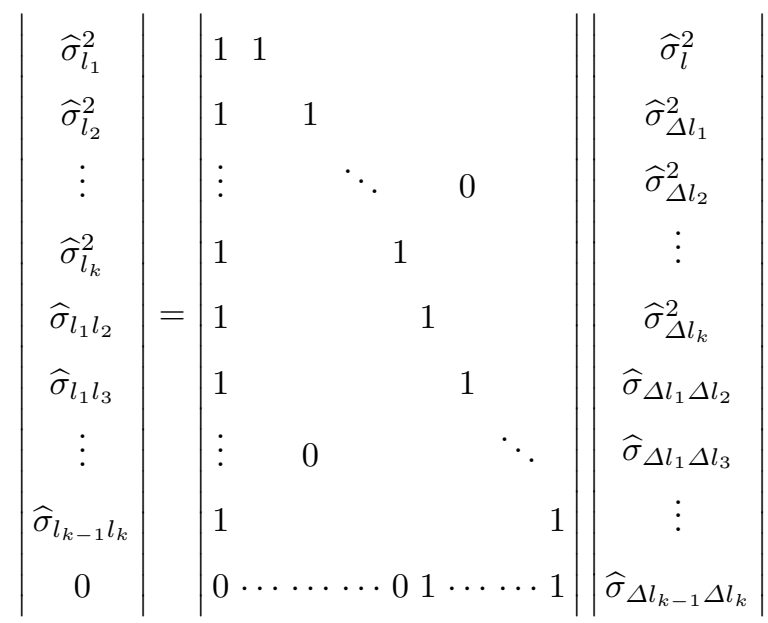

which includes the additional restriction equation, $\sum \widehat{\sigma}_{\Delta l_{i} \Delta l_{j}}=0$, in the last line to prevent rank deficiency (Fang 2007). The elements of the above matrix can be estimated through

$\widehat{\sigma}_{l_{i}}^{2}=\frac{1}{u-1} \sum\left(l_{i}-\text { mean }\left(l_{i}\right)\right)^{2}$ and $\widehat{\sigma}_{l_{i} l_{j}}=\frac{1}{u-1} \sum\left(l_{i}-\right.$ mean $\left.\left(l_{i}\right)\right)\left(l_{j}-\right.$ mean $\left.\left(l_{j}\right)\right)$

where the mean $(\cdots)$ operator denotes the arithmetic mean of the elements of the vector in brackets. The hat above the vector, $\widehat{\sigma}$, enables distinguishing between the empirical estimates of variances and covariances used here and the formal errors, $\sigma$, reported by the ACs. Furthermore, the stochastic model is extended by off-diagonal elements assuming pairwise uncorrelated vectors $l$ and $\Delta l_{i}$ :

$Q_{l_{i} l_{j}}=\left|\begin{array}{lll}I & I & 0\end{array}\right|\left|\begin{array}{ccc}Q_{l l} & 0 & 0 \\ 0 & Q_{\Delta l_{i} \Delta l_{i}} & 0 \\ 0 & 0 & Q_{\Delta l_{j} \Delta l_{j}}\end{array}\right|\left|\begin{array}{l}I \\ 0 \\ I\end{array}\right|=Q_{l_{j} l_{i}}=Q_{l l}$ 
yielding a cofactor matrix with block-diagonal structure composed of a common part due to the identical original observations and an individual part accounting for the analysis noise of the ACs:

$$
Q_{\overline{l l}}=\left|\begin{array}{cccc}
Q_{l l} & Q_{l l} & \cdots & Q_{l l} \\
Q_{l l} & Q_{l l} & \ddots & \vdots \\
\vdots & \ddots & \ddots & Q_{l l} \\
Q_{l l} & \cdots & Q_{l l} & Q_{l l}
\end{array}\right|+\left|\begin{array}{cccc}
Q_{\Delta l_{1} \Delta l_{1}} & 0 & \cdots & 0 \\
0 & Q_{\Delta l_{2} \Delta l_{2}} & \ddots & \vdots \\
\vdots & \ddots & \ddots & 0 \\
0 & \cdots & 0 & Q_{\Delta l_{k} \Delta l_{k}}
\end{array}\right|
$$

According to Kutterer et al. (2009) the following approach with one global correction factor can be used:

$Q_{\Delta l_{i} \Delta l_{i}}=\bar{\alpha}^{2} \cdot Q_{l l}$

modeling a global analysis noise level, $\bar{\alpha}^{2}$, common to all ACs.

\subsection{Combination results}

The variance components (VCs) estimated with model (equ. 7) exhibit large differences (tab. 6) among ACs. Since the VCs are estimated considering the reported formal errors, they can not be interpreted independent of the reported errors. Thus, besides the VCs, table (6) holds the product of the square root of the VCs with the mean values of the reported formal errors of the individual AC. This product is the actual square root of the cofactors considered in the model with variance components. The products show large differences, e.g. between 4.71 (DGFI) and 20.74 (MAO) at Concepción. AUS, IAA, $\mathrm{MAO}$, and OSO show larger products of mean formal errors with the square root of the VCs, which coincide with the empirically assessed mean offsets and standard deviations (sec. 3). The products also differ from station to station: no AC shows smallest or largest values at all stations. Since VCs of AUS are in the order of 10, the empirically determined formal errors of AUS are about sqrt(10) times too optimistic. The last column in table (6) holds the mean value of the mean formal errors of the ACs and the mean values of the product of the mean formal errors and the square root of the VCs. Since the products are about twice as large as the mean formal errors, the reported formal errors are too optimistic in general.

Figure (7, left) shows the mean difference of $Z T D$ s of all stations applying the stochastic combination models with regard to the diagonal model (equ. 6). All four models of parameter combination provide equivalent results in terms of the parameters. Mean parameter differences stay below $0.1 \mathrm{~mm}$. The combined parameters themselves show only very small differences, but the different stochastic models significantly affect the formal errors of the combined parameters, which is illustrated in figure (7, 
right), where the relation of the mean of the formal errors of all stations with respect to the diagonal model show large differences. While the unweighted combination provides the smallest formal errors, about $50 \%$ to $60 \%$ of the size of the mean formal errors of the diagonal model, the model with variance components and the OSI model are up to twice as large as the corresponding mean formal errors of the diagonal model.

Table (7) summarizes the relevant results. The common OSI parameters, $\bar{\alpha}^{2}$, vary between 2.24 (Medicina) and 3.29 (Zelenchukskaya) with a mean value of 2.65. They give the ratio of the analysis to the observation noise. In the case of $Z T D$ s the analyses add noise of about 2.5 times the size of the observational noise. At least for Zelenchukskaya the larger analysis noise is probably caused by the different treatment of surface pressure.

The mean formal errors of the combination models (tab.7) are derived from the final standard deviations of the combined ZTDs. The unweighted combination model produces unrealistically small mean formal errors $(2.34 \mathrm{~mm})$. Using the reported formal errors in the diagonal model results in formal errors of the combined parameters of $4.37 \mathrm{~mm}$, about the same size as the empirical mean standard deviation of reported $Z T D(4.6 \mathrm{~mm})$ derived in sec. (3). Considering individual VCs in addition to the reported formal errors yields about twice as large formal errors $(8.60 \mathrm{~mm})$. The reapplication of observations considered in the OSI model again yields about twice as large formal errors (8.60 $\mathrm{mm})$ indicating that neglecting the usage of the same observations leads to too optimistic errors.

\section{Summary and conclusion}

(i) Despite recommendations, different methods are in use for the substitution of missing pressure values. The inconsistent application of surface air pressure data in VLBI analyses causes the largest systematic effect on estimated zenith delays. Since the reported $Z W D$ s reflect the inconsistent usage of meteorological data, the combined $Z W D$ s are not derived by a combination of the reported $Z W D$ s. The $Z T D$ s, which depend much less on pressure inconsistencies, are combined and one consistent series of $Z H D \mathrm{~s}$, taken from the DGFI solution, is subtracted for the derivation of consistent combined $Z W D$ s. The reported gradients do not allow for a meaningful combination. The conventions on the analysis options have to include a parameterization of the gradients before these troposphere parameters can be combined. In addition the weights of the gradient constraints have to be assessed in order to make the adjusted gradients independent from the apriori gradients. An appropriate apriori model for total atmosphere gradients, such as the DAO gradients (MacMillan and Ma 1998) should provide the basis for a more precise gradient determination.

(ii) Although the most relevant analysis options were conventionally harmonized, significant differences 
between IVS AC solutions can be found for some sites. A combination of parameters based on different analysis options without considering the effects would have two major problems: the combination would be ambiguous, i.e. it would not be possible to specify a unique model for the combination, and it would be inconsistent, i.e. systematic differences would be present among the individual solutions leading to systematic errors of the combination. A consistent combination requires correcting all significant effects. The sequential variation of models allows the determination of model impacts, which can be used to empirically correct inconsistent model usage among the ACs to a certain extent. The empirically corrected contribution of CGS obtains the smallest VC at some sites, which shows that the differences between the usage of NMF and VMF1 can be successfully corrected with such an approach. Due to the correlations of troposphere parameters with other groups of parameters, the empirical corrections determined in that way are only admissible if these correlations stay small. Thus, the application of the same models is still the only way to ensure consistent results. Apart from models, the impact of parameterizations and constraints must be assessed together in one step. Simulating the parameterizations and constraints of other ACs using the Gauss-Markov-model again implicitly neglects the correlations with other parameter groups. The various methods of data editing can cause further significant effects: AUS and IAA contributions, for example, show significantly larger standard deviations compared to the other ACs. The downweighting of low elevation observations may be a reason for the increased standard deviations and the relative large VCs. The application of the random walk model for zenith delays may be another reason for larger deviations, which is also observable for the contribution of MAO. Some of the observed significant differences could not be explained by the usage of the considered analysis options. Those remaining differences are probably due to different modeling of other correlated parameters outside of the troposphere model, such as the clock parameters.

(iii) If no reference model is available, the comparison between more than two solutions provides the only way to define and detect outliers. Increasing the number of solutions that are included for comparison, will increase the significance with which outliers can be identified. The outlier treatment is the analysis step which leads to robustness (Kutterer et al. 2003), not to reliability (Kutterer 2004), which is often incorrectly referred to in this context. The comparison provides robustness, not the combination. If the combination is done including unconsidered outliers, the combined result will consist of a mixture of observations and outliers, which may mask the actual outliers and thus make it more difficult or even impossible to detect the outliers afterwards.

(iv) The intra-technique combination works on the same observations (reapplication of observations), hence, its usefulness is not immediately evident and its application has to be justified. The reasons working with intra-technique combinations are not obvious from a scientific point of view because the usage of the same original observations does not add new information to the equation system, 
it only reweights the observations. The same observations are analyzed by various ACs in different ways adding more analysis noise than probably necessary. As the determined common noise parameters, $\bar{\alpha}^{2}$, show, the analysis noise is about two and a half times larger than the observation noise. Thus, the analysis noise is the dominant noise contributor. Standards, such as the IERS conventions, specify state-of-the-art models to be used for analysis because the models were found to be superior. Parameterizations are not standardized in the way models are, and it is theoretically and empirically not simple to determine the optimal parameterization. If constraints are to be applied, a variety of possibilities exist to define what type and size of constraints are used. In principle, there are theoretical and empirical ways to find optimal constraints. The same holds for the data editing methods, which can be theoretically compared but very often rely on empirically found threshold values. If a mixture of all these analysis options is combined, it will be difficult to interpret the characteristics of the combination. In particular, a combination based on various models, e.g. an obsolete and a new model, is not desirable. In addition, the intra-technique combination procedure can be another possible error source, if incorrectly performed. Most intra-technique combination algorithms neglect the reapplication of observations and other correlations among the input data and thus provide formal errors that are unrealistic. Nevertheless intra-technique combinations are applied to generate most of the official products of IVS, including the troposphere parameters.

(v) The formal errors of the more realistic combinations show that the reported formal errors are too optimistic. One reason for the too small formal errors could be the neglect of the accuracy of the surface pressure data. If an additional noise level due to the estimated pressure accuracy ( $2 \mathrm{hPa})$ of $4 \mathrm{~mm}$ is considered in addition (sec. 3.1.1), the resulting uncertainty of $8.4 \mathrm{~mm}$ gets much closer to the mean formal errors of $8.6 \mathrm{~mm}$ derived by the more realistic combination models.

(vi) Identical observations are repeatedly used for every intra-technique combination, thus, the combination model should consider this. The inclusion of off-diagonal cofactors modeling the reapplication of observations in the OSI model does not significantly affect the combined parameters, but in the case of $Z T D \mathrm{~s}$ results in different mean formal errors scaled by a factor of about two with regard to the diagonal model. The sizes of the estimated VCs show both, that the reported formal errors of the ACs are too optimistic and that the reported formal errors have to be scaled with regard to each other. The necessity of scaling can arise from the inconsistent analysis options. The various estimation techniques (Gauss-Markov-model, filter, and collocation techniques), however, certainly also contribute to this effect. The actual effects of the estimation technique on the formal errors should be investigated. Since the determined VCs differ significantly, the assumption of a common noise level of all ACs has to be reconsidered. The variance component model also produces formal errors scaled by a factor of about two with regard to the diagonal model. Thus, it is necessary to consider both approaches, the scaling 
(VC estimation) and the reapplication of observations (OSI model). Such a model should be developed and applied for intra-technique combination in future.

(vii) As shown by Steinforth and Nothnagel (2004) for other parameters, correlations between different groups of estimated troposphere parameters, e.g. between gradients and zenith delays, can occur in addition during parameter estimation. These inter-parameter correlations are to be considered in the future as well. On the level of parameter estimates this is impossible; therefore a combination on the level of normal equations is necessary and recommended.

Concluding, CONT08 VLBI observations provide a very valuable data set for the determination of precise troposphere parameters. The high quality and density of observations allows better estimates of the troposphere parameters and more significant statements on the characteristics of the troposphere. For the combination of troposphere parameters a combination of variance component estimation and the OSI model is recommended.

Acknowledgements We acknowledge the IVS (Schlüter and Behrend 2007) and its components for providing excellent VLBI data. J. Böhm would like to thank the FWF for supporting this work within project P20902 (GGOS Atmosphere). The authors thank the three reviewers, whose comments helped improve the article.

\section{References}

Altamimi Z, Sillard P, Boucher C (2002) ITRF2000: A new release of the International Terrestrial Reference Frame for Earth Science Applications. J Geophys Res 107(B10), 2214, doi:10.1029/2001JB000561

Altamimi Z, Collilieux X, Legrand J, Garayt B, Boucher C (2007) ITRF2005: A new release of the International Terrestrial Reference Frame based on time series of station positions and Earth orientation parameters. J Geophys Res 112(B09401), doi:10.1029/2007JB004949

Angermann D, Drewes H, Kruegel M, Meisel B, Gerstl M, Kelm R, Mueller H, Seemueller W, Tesmer V (2004) ITRS Combination Center at DGFI: A Terrestrial Reference Frame Realization 2003. Verlag der Bayerischen Akademie der Wissenschaften in Kommission beim Verlag C. H. Beck, Reihe B (313), München Berg H (1948) Allgemeine Meteorologie. Dümmler, Bonn

Bierman GJ (1977) Factorization Methods for Discrete Sequential Estimation. Mathematics in Science and Engineering 128, Bellman R (ed.), University of Southern California

Böckmann S, Artz T, Nothnagel A (2010) VLBI Terrestrial reference frame contributions to ITRF2008. J Geod(2010) 84:201-219, doi:10.1007/s00190-009-0357-7

Böhm J, Messerer E, Schuh H (2002) Comparison of Tropospheric Parameters Submitted to the IVS Analysis Pilot Project. IVS 2002 General Meeting Proceedings, Vandenberg NR and Baver KD (eds.), NASA/CP2002-210002, 340-344

Böhm J, Werl B, Schuh H (2006) Troposphere mapping functions for GPS and VLBI from ECMWF operational analysis data. J Geophys Res 111(B02406), doi:10.1029/2005JB003629

Böhm J, Heinkelmann R, Schuh H (2007) Short note: a global model of pressure and temperature for geodetic applications. J Geod 81(10):679-683 
Chen G, Herring TA (1997) Effects of atmospheric azimuthal asymmetry on the analysis of space geodetic data, J Geophys Res 102:20,489-20,502

Davis J, Herring TA, Shapiro II, Rogers AEE, Elgered G (1985) Geodesy by Radio Interferometry: Effects of atmospheric modelling errors on the estimates of baseline lengths, Rad Sci 20:1593-1607

Elgered G (2001) An overview of COST Action 716: exploitation of ground-based GPS for climate and numerical weather prediction applications, Phys Chem Earth 26(6-8):399-404

Fang X (2007) Statistische Analyse von Ausgleichungsergebnissen auf der Basis teilweise identischer Messwerte. Diplomarbeit im Studiengang Geodäsie und Geoinformatik, Geodätisches Institut, Leibniz Universität Hannover

Förstner W (1979) Ein Verfahren zur Schätzung von Varianz- und Kovarianzkomponenten. Allg Vermess-Nachr (11-12):446-453

Gelb A (ed.) (1974) Applied optimal estimation. Sixth printing, The M.I.T. Press, Massachusetts Institute of Technology, Cambridge, Massachusetts, and London, England

Heinkelmann R (2008) Bestimmung des atmosphärischen Wasserdampfes mittels VLBI als Beitrag zur Klimaforschung. Geowissenschaftliche Mitteilungen No. 82, ISSN 1811-8380

Heinkelmann R (2009) IVSTrop: Status and recommendations of the IVS rapid troposphere combination. Proceedings of the 19th EVGA Working Meeting. Bourda G, Charlot P, and Collioud A (eds.), Université Bordeaux 1 - CNRS, Observatoire Aquitain des Sciences de l'Univers, Laboratoire d'Astrophysique de Bordeaux, 180-182

Heinkelmann R, Böhm J, Schuh H (2005) Homogenization of surface pressure recordings and its impact on longterm series of VLBI tropospheric parameters. Proceedings of the 17th EVGA Working Meeting. Vennebusch M. and Nothnagel A. (eds.), INAF - Istituto di Radioastronomia - Sezione di NOTO - Italy, 74-78

Heinkelmann R, Böhm J, Schuh H, Bolotin S, Engelhardt G, MacMillan DS, Negusini M, Skurikhina E, Tesmer V, Titov O (2007) Combination of long time-series of troposphere zenith delays observed by VLBI. J Geod 81(6-8):483-501

Heinkelmann R, Böhm J, Schuh H, Tesmer V (2009) The influence of meteorological input data on the VLBI reference frames. Drewes H (ed.) Geodetic Reference Frames, International Association of Geodesy Symposium 134, Springer Verlag Berlin Heidelberg:245-251

IERS (2004) IERS Conventions (2003), McCarthy DD and Petit G (eds.), IERS Technical Note No. 32, Verlag des Bundesamts für Kartographie und Geodäsie, Frankfurt am Main

Jin S, Wu Y, Heinkelmann R, Park J (2008) Diurnal and semidiurnal atmospheric tides observed by co-located GPS and VLBI measurements. Journal of Atmospheric and Solar-Terrestrial Physics, Vol. 70 (2008):13661372

Koch KR (1997) Parameterschätzung und Hypothesentests, 3rd edition. Dümmler, Bonn

Kusche J (2003) A Monte-Carlo technique for weight estimation in satellite geodesy. J Geod 76:641-652

Kutterer H (2004) Reliability measures for geodetic VLBI products. IVS 2004 General Meeting Proceedings, Vandenberg NR and Baver KD (eds), NASA/CP-2004-212255, 301-305

Kutterer H, Heinkelmann R, Tesmer V (2003) Robust outlier detection in VLBI data analysis. Proceedings of the 16th EVGA Working Meeting. Schwegmann W and Thorandt V (eds.), Verlag des Bundesamts für Kartographie und Geodäsie, Leipzig Frankfurt am Main, 247-256

Kutterer H, Krügel M, Tesmer V (2009) Towards an Improved Assessment of the Quality of Terrestrial Reference Frames. Geodetic Reference Frames. Drewes H (ed.), International Association of Geodesy Symposia 
134, doi:10.1007/978-3-642-00860-3_ 10, Springer Verlag Berlin Heidelberg:67-72

MacMillan DS (1995) Atmospheric gradients from very long baseline interferometry observations. Geophys Res Let 22(9):1041-1044

MacMillan DS, Ma C (1998) Using meteorological data assimilation models in computing tropospheric delays at microwave frequencies. Phys Chem Earth, Vol. 23, No. 1, 97-102

Niell AE (1996) Global mapping functions for the atmosphere delay at radio wavelength, J Geophys Res 101(B2):3227-3246

Nothnagel A, Vennebusch M, Campbell J (2002) On correlations between parameters in geodetic VLBI data analysis. IVS 2002 General Meeting Proceedings, Vandenberg NR and Baver KD (eds.), NASA/CP-2002210002, 260-264

Plank L, Böhm J, Schuh H (2010) Comparison Campaign of VLBI data analysis software - first results. IVS 2010 General Meeting Proceedings, Behrend D and Baver KD (eds.), in press

Schuh H, Böhm J (2003) IVS Pilot Project - Tropospheric Parameters. Österreichische Zeitschrift für Vermessung und Geoinformation VGI(91) 01:14-20

Schlüter W, Behrend D (2007) The International VLBI Service for Geodesy and Astrometry (IVS): current capabilities and future prospects. J Geod 81(6-8):379-387

Steinforth C, Nothnagel A (2004) Considering a Priori Correlations in the IVS Combined EOP Series. IVS 2004 General Meeting Proceedings, Vandenberg NR and Baver KD (eds.), NASA/CP-2004-212255, 413-417

Teke K, Böhm J, Nilsson T, Schuh H, Steigenberger P, Dach R, Heinkelmann R, Willis P, Haas R, Garcia Espada S, Hobiger T, Ichikawa R, Shimizu S (this issue) Multi-technique comparison of troposphere zenith delays and gradients during CONT08. J Geod, submitted

Tesmer V (2004) Das stochastische Modell bei der VLBI-Auswertung. DGK Reihe C, Dissertationen, Heft Nr. 573, ISBN 376965012 3, Verlag der Bayerischen Akademie der Wissenschaften in Kommission beim Verlag C. H. Beck, München, 105

Titov O, Schuh H (2000) Short periods in Earth rotation seen in VLBI data analysed by the least-squares collocation method. IERS Technical Note No. 28, Observatoire de Paris, Paris, 33-41 
Table 1 The ten IVS Analysis Centers contributing input data to this special issue

\begin{tabular}{|l|l|}
\hline IVS AC & institution \\
\hline AUS & Geoscience Australia, Canberra, Australia \\
BKG & Bundesamt für Kartographie und Geodäsie, Leipzig, Germany \\
CGS & Centro di Geodesia Spaziale, Matera, Italy \\
DGFI & Deutsches Geodätisches Forschungsinstitut, Munich, Germany \\
GSFC & NASA Goddard Space Flight Center, Greenbelt, USA \\
IAA & Institute of Applied Astronomy, St. Petersburg, Russia \\
IGG & Institute of Geodesy and Geophysics, Vienna, Austria \\
INA & Istituto di Radioastronomia, Istituto Nazionale di Astrofisica, Bologna, Italy \\
MAO & Main Astronomical Observatory, National Academy of Sciences of Ukraine, Kiev, Ukraine \\
OSO & Onsala Space Observatory, Chalmers University of Technology, Onsala, Sweden \\
\hline
\end{tabular}

Table 2 Relevant models and meteorological data used by the ten contributing IVS Analysis Centers. Acronyms are explained in the text. $(*)$ : At first the DGFI solution was provided using non-zero apriori gradients (MacMillan and Ma 1998). For the sake of consistency a solution with zero apriori gradients was computed and used for the combination.

\begin{tabular}{l|c|c|c|c|}
\hline AC & $\begin{array}{c}\text { TRF } \\
\text { a priori }\end{array}$ & $\begin{array}{c}\text { mapping } \\
\text { functions }\end{array}$ & $\begin{array}{c}\text { gradients } \\
\text { a priori }\end{array}$ & $\begin{array}{c}\text { substitute for } \\
\text { local met data }\end{array}$ \\
\hline AUS & ITRF2000 & VMF1 & zero & GPT model (Böhm et al. 2007) \\
\hline BKG & VTRF2008 & VMF1 & zero & standard model (Berg 1948) \\
\hline CGS & ITRF2000 & NMF & zero & standard model (Berg 1948) \\
\hline DGFI & VTRF2008 & VMF1 & zero (*) & numerical weather model \\
\hline GSFC & VTRF2008 & VMF1 & zero & numerical weather model \\
\hline IAA & VTRF2008 & VMF1 & zero & numerical weather model \\
\hline IGG & ITRF2005 & VMF1 & zero & GPT model (Böhm et al. 2007) \\
\hline INA & ITRF2000 & NMF & zero & standard model (Berg 1948) \\
\hline MAO & ITRF2000 & NMF & zero & GPT model (Böhm et al. 2007) \\
\hline OSO & VTRF2008 & NMF & zero & standard model (Berg 1948) \\
\hline
\end{tabular}


Table 3 Troposphere parameterization and constraints of the ten contributing IVS Analysis Centers. Acronyms are explained in the text. Only the constraints of the ACs using the Gauss-Markov-model (GMM) are given. Although the stochastic model of the RW-process applied with the filter or collocation techniques affects the estimates in a way similar to the constraints of the rate relative to zero used in the GMM, the values cannot be compared directly and, thus, are omitted.

\begin{tabular}{|c|c|c|c|c|}
\hline \multirow[t]{2}{*}{$\mathrm{AC}$} & \multicolumn{2}{|c|}{ zenith delays } & \multicolumn{2}{|c|}{ gradients } \\
\hline & parameterization & constraints & parameterization & contraints \\
\hline AUS & 1h-mean of RW & - & 1h-mean of RW & - \\
\hline $\mathrm{BKG}$ & 1h-PWLF & $\begin{array}{c}\text { offset: - } \\
\text { rate: } 15 \mathrm{~mm} / \mathrm{h}\end{array}$ & $\begin{array}{l}24 \mathrm{~h} \text {-offset } \\
24 \mathrm{~h} \text {-rate }\end{array}$ & $\begin{array}{l}\text { offset: } 0.5 \mathrm{~mm} \\
\text { rate: } 2.0 \mathrm{~mm} / 24 \mathrm{~h}\end{array}$ \\
\hline CGS & 1h-PWLF & $\begin{array}{c}\text { offset: - } \\
\text { rate: } 15 \mathrm{~mm} / \mathrm{h}\end{array}$ & $\begin{array}{l}\text { 3h-offset } \\
\text { 3h-rate }\end{array}$ & $\begin{array}{l}\text { offset: } 5.0 \mathrm{~mm} \\
\text { rate: } 2.0 \mathrm{~mm} / 24 \mathrm{~h}\end{array}$ \\
\hline DGFI & 1h-PWLF & $\begin{array}{l}\text { offset: - } \\
\text { rate: } 10 \mathrm{~mm} / \mathrm{h}\end{array}$ & $\begin{array}{l}24 \mathrm{~h} \text {-offset } \\
24 \mathrm{~h} \text {-rate }\end{array}$ & $\begin{array}{l}\text { offset: } 0.5 \mathrm{~mm} \\
\text { rate: } 2.0 \mathrm{~mm} / 24 \mathrm{~h}\end{array}$ \\
\hline GSFC & 1h-PWLF & $\begin{array}{c}\text { offset: - } \\
\text { rate: } 15 \mathrm{~mm} / \mathrm{h}\end{array}$ & $\begin{array}{l}\text { 8h-offset } \\
\text { 8h-rate }\end{array}$ & $\begin{array}{l}\text { offset: } 0.5 \mathrm{~mm} \\
\text { rate: } 2.0 \mathrm{~mm} / 24 \mathrm{~h}\end{array}$ \\
\hline IAA & 1h-mean of RW & - & 1h-mean of RW & - \\
\hline IGG & 1h-averaged PWLF & $\begin{array}{l}\text { offset: - } \\
\text { rate: } 15 \mathrm{~mm} / \mathrm{h}\end{array}$ & 2h-PWLF & $\begin{array}{l}\text { offset: - } \\
\text { rate: } 2.0 \mathrm{~mm} / 24 \mathrm{~h}\end{array}$ \\
\hline INA & 1h-PWLF & $\begin{array}{l}\text { offset: - } \\
\text { rate: } 15 \mathrm{~mm} / \mathrm{h}\end{array}$ & $\begin{array}{l}\text { 24h-offset } \\
24 \text {-rate }\end{array}$ & $\begin{array}{l}\text { offset: } 0.5 \mathrm{~mm} \\
\text { rate: } 2.0 \mathrm{~mm} / 24 \mathrm{~h}\end{array}$ \\
\hline MAO & 1h-mean of RW & - & 1h-mean of RW & - \\
\hline OSO & 1h-PWLF & $\begin{array}{l}\text { offset: - } \\
\text { rate: } 6 \mathrm{~mm} / \mathrm{h}\end{array}$ & $\begin{array}{l}\text { 6h-offset } \\
\text { 6h-rate }\end{array}$ & $\begin{array}{l}\text { offset: } 2.0 \mathrm{~mm} \\
\text { rate: } 2.0 \mathrm{~mm} / 24 \mathrm{~h}\end{array}$ \\
\hline
\end{tabular}

Table 4 Data editing and reweighting of the ten contributing IVS Analysis Centers. Acronyms are explained in the text. Since there are no observations performed below $5^{\circ}$ elevation during CONT08, the differing cutoff elevation angles are irrelevant.

\begin{tabular}{|c|c|c|c|}
\hline $\mathrm{AC}$ & $\begin{array}{c}\text { cutoff elevation } \\
\text { angle }\end{array}$ & $\begin{array}{l}\text { downweighting } \\
\text { of low observ. }\end{array}$ & $\begin{array}{l}\text { reweihting } \\
\text { of observ. }\end{array}$ \\
\hline AUS & $5^{\circ}$ & $\cos \left(90^{\circ}-\epsilon\right)$ below $10^{\circ}$ & - \\
\hline $\mathrm{BKG}$ & $5^{\circ}$ & - & - \\
\hline CGS & $3^{\circ}$ & - & - \\
\hline DGFI & - & - & Tesmer (2004) \\
\hline GSFC & $3^{\circ}$ & - & - \\
\hline IAA & - & $\cos ^{2}\left(90^{\circ}-\epsilon\right)$ below $10^{\circ}$ & - \\
\hline IGG & - & - & - \\
\hline INA & $5^{\circ}$ & - & - \\
\hline MAO & - & - & - \\
\hline $\mathrm{OSO}$ & $5^{\circ}$ & - & - \\
\hline
\end{tabular}


Table 5 Empirical statistics derived through comparisons of the input series. Station-wise standard deviations of the constant offsets of the ACs with regard to the unweighted mean ('bias') and station-wise mean values of the empirical standard deviations of the ACs with regard to the unweighted mean ('stdev'). The last row ('mean') gives the mean of the values in the above columns.

\begin{tabular}{|l|r|r|r|r|r|r|r|r|}
\hline IVS site & \multicolumn{2}{|c|}{$Z T D$} & \multicolumn{2}{|c|}{$Z W D$} & \multicolumn{2}{|c|}{$G_{N}$} & \multicolumn{2}{|c|}{$G_{E}$} \\
& bias & stdev & bias & stdev & bias & stdev & bias & stdev \\
\hline HARTRAO & 1.8 & 5.5 & 1.9 & 5.5 & 0.3 & 0.5 & 0.1 & 0.5 \\
KOKEE & 2.1 & 4.9 & 2.3 & 4.7 & 0.2 & 0.5 & 0.1 & 0.4 \\
MEDICINA & 3.6 & 4.5 & 3.7 & 4.5 & 0.2 & 0.4 & 0.1 & 0.3 \\
NYALES20 & 2.6 & 2.4 & 2.5 & 2.5 & 0.1 & 0.3 & 0.1 & 0.3 \\
ONSALA60 & 3.7 & 2.9 & 3.9 & 2.9 & 0.1 & 0.3 & 0.1 & 0.3 \\
SVETLOE & 3.5 & 4.3 & 6.3 & 3.9 & 0.1 & 0.3 & 0.1 & 0.3 \\
TIGOCONC & 6.9 & 5.4 & 6.7 & 5.1 & 0.2 & 0.4 & 0.2 & 0.4 \\
TSUKUB32 & 3.8 & 7.2 & 4.2 & 6.2 & 0.2 & 0.6 & 0.1 & 0.5 \\
WESTFORD & 3.0 & 4.5 & 3.0 & 4.6 & 0.1 & 0.5 & 0.1 & 0.4 \\
WETTZELL & 3.1 & 2.8 & 3.1 & 2.8 & 0.1 & 0.2 & 0.1 & 0.3 \\
ZELENCHK & 3.6 & 6.2 & 6.0 & 6.9 & 0.3 & 0.6 & 0.1 & 0.5 \\
\hline mean & 3.4 & 4.6 & 4.0 & 4.5 & 0.2 & 0.4 & 0.1 & 0.4 \\
\hline
\end{tabular}


Table 6 Variance components $\left(V C_{i}\right)$ and mean values of the reported formal errors of $Z T D \mathrm{~s}\left(\sigma_{i}\right)$ in mm. The product of the square root of the $\mathrm{VC}$ and the corresponding mean formal error $\left(\operatorname{mean}\left(\sigma_{i}\right) \cdot \sqrt{V C_{i}}\right)$ equals the actual square root of the cofactor determined by the variance component estimation.

\begin{tabular}{|c|c|c|c|c|c|c|c|c|c|c|c|}
\hline ZTD & AUS & BKG & CGS & DGFI & GSFC & IAA & IGG & INA & MAO & OSO & mean \\
\hline \multicolumn{12}{|c|}{ HARTRAO (Hartebeesthoek, South Africa) } \\
\hline$V C_{i}$ & 10.33 & 2.90 & 2.75 & 0.94 & 2.35 & 2.44 & 2.32 & 12.79 & 14.48 & 12.69 & - \\
\hline $\operatorname{mean}\left(\sigma_{i}\right)$ & 2.62 & 3.53 & 3.29 & 6.15 & 3.44 & 8.33 & 3.24 & 3.32 & 2.90 & 3.01 & 3.98 \\
\hline $\operatorname{mean}\left(\sigma_{i}\right) \cdot \sqrt{V C_{i}}$ & 8.42 & 6.01 & 5.46 & 5.96 & 5.27 & 13.01 & 4.94 & 11.87 & 11.04 & 10.72 & 8.27 \\
\hline \multicolumn{12}{|c|}{ KOKEE (Kokee Park, Kauai, Hawaii) } \\
\hline$V C_{i}$ & 8.09 & 7.01 & 2.69 & 1.45 & 5.30 & 3.51 & 4.70 & 8.08 & 13.52 & 11.84 & - \\
\hline $\operatorname{mean}\left(\sigma_{i}\right)$ & 4.43 & 2.01 & 2.42 & 4.15 & 2.71 & 5.70 & 2.13 & 2.58 & 2.48 & 2.42 & 3.10 \\
\hline $\operatorname{mean}\left(\sigma_{i}\right) \cdot \sqrt{V C_{i}}$ & 12.60 & 5.32 & 3.97 & 5.00 & 6.24 & 10.68 & 4.62 & 7.33 & 9.12 & 8.33 & 7.32 \\
\hline \multicolumn{12}{|c|}{ MEDICINA (Bologna, Italy) } \\
\hline$V C_{i}$ & 5.84 & 8.82 & 8.17 & 3.32 & 5.19 & 2.85 & 4.03 & 11.04 & 34.85 & 21.19 & - \\
\hline $\operatorname{mean}\left(\sigma_{i}\right)$ & 3.94 & 1.87 & 1.76 & 2.52 & 2.09 & 5.07 & 1.87 & 1.73 & 1.73 & 1.82 & 2.44 \\
\hline $\operatorname{mean}\left(\sigma_{i}\right) \cdot \sqrt{V C_{i}}$ & 9.52 & 5.55 & 5.03 & 4.59 & 4.76 & 8.56 & 3.75 & 5.75 & 10.21 & 8.38 & 6.61 \\
\hline \multicolumn{12}{|c|}{ NYALES20 (Ny-Ålesund, Spitsbergen, Norway) } \\
\hline$V C_{i}$ & 3.38 & 3.46 & 0.56 & 1.39 & 2.47 & 2.03 & 2.69 & 3.76 & 9.29 & 7.31 & - \\
\hline $\operatorname{mean}\left(\sigma_{i}\right)$ & 2.50 & 1.97 & 1.64 & 3.20 & 2.02 & 4.91 & 1.91 & 1.60 & 1.77 & 1.73 & 2.33 \\
\hline $\operatorname{mean}\left(\sigma_{i}\right) \cdot \sqrt{V C_{i}}$ & 4.60 & 3.66 & 1.23 & 3.77 & 3.17 & 7.00 & 3.13 & 3.10 & 5.39 & 4.68 & 3.97 \\
\hline \multicolumn{12}{|c|}{ ONSALA60 (Onsala, Sweden) } \\
\hline$V C_{i}$ & 4.61 & 4.07 & 1.35 & 1.18 & 3.44 & 3.04 & 3.21 & 2.94 & 17.56 & 11.70 & - \\
\hline $\operatorname{mean}\left(\sigma_{i}\right)$ & 3.34 & 1.77 & 1.59 & 2.61 & 1.91 & 4.41 & 1.77 & 1.58 & 1.67 & 1.61 & 2.23 \\
\hline $\operatorname{mean}\left(\sigma_{i}\right) \cdot \sqrt{V C_{i}}$ & 7.17 & 3.57 & 1.85 & 2.84 & 3.54 & 7.69 & 3.17 & 2.71 & 7.00 & 5.51 & 4.50 \\
\hline \multicolumn{12}{|c|}{ SVETLOE (St. Petersburg, Russia) } \\
\hline$V C_{i}$ & 6.05 & 3.89 & 4.06 & 3.77 & 2.94 & 1.74 & 2.98 & 4.23 & 30.43 & 11.05 & - \\
\hline $\operatorname{mean}\left(\sigma_{i}\right)$ & 3.72 & 2.23 & 1.86 & 2.66 & 2.12 & 5.23 & 2.18 & 1.88 & 1.70 & 2.11 & 2.57 \\
\hline $\operatorname{mean}\left(\sigma_{i}\right) \cdot \sqrt{V C_{i}}$ & 9.15 & 4.40 & 3.75 & 5.16 & 3.64 & 6.90 & 3.76 & 3.87 & 9.38 & 7.01 & 5.70 \\
\hline \multicolumn{12}{|c|}{ TIGOCONC (Concepción, Chile) } \\
\hline$V C_{i}$ & 10.06 & 2.80 & 1.34 & 0.52 & 2.19 & 3.02 & 3.27 & 9.01 & 23.37 & 17.34 & - \\
\hline $\operatorname{mean}\left(\sigma_{i}\right)$ & 3.27 & 4.00 & 4.16 & 6.53 & 4.25 & 7.93 & 3.64 & 4.44 & 4.29 & 4.27 & 4.68 \\
\hline $\operatorname{mean}\left(\sigma_{i}\right) \cdot \sqrt{V C_{i}}$ & 10.37 & 6.69 & 4.82 & 4.71 & 6.29 & 13.78 & 6.58 & 13.33 & 20.74 & 17.78 & 10.51 \\
\hline \multicolumn{12}{|c|}{ TSUKUB32 (Tsukuba, Japan) } \\
\hline$V C_{i}$ & 15.74 & 10.45 & 2.65 & 1.86 & 6.59 & 11.42 & 7.87 & 12.96 & 34.05 & 21.71 & - \\
\hline $\operatorname{mean}\left(\sigma_{i}\right)$ & 4.06 & 2.13 & 2.25 & 5.67 & 2.62 & 5.79 & 2.09 & 2.32 & 2.28 & 2.40 & 3.16 \\
\hline $\operatorname{mean}\left(\sigma_{i}\right) \cdot \sqrt{V C_{i}}$ & 16.11 & 6.89 & 3.66 & 7.73 & 6.73 & 19.57 & 5.86 & 8.35 & 13.30 & 11.18 & 9.94 \\
\hline \multicolumn{12}{|c|}{ WESTFORD (Haystack, USA) } \\
\hline$V C_{i}$ & 5.45 & 6.70 & 3.86 & 2.07 & 3.86 & 4.20 & 3.17 & 9.07 & 19.56 & 12.44 & - \\
\hline $\operatorname{mean}\left(\sigma_{i}\right)$ & 3.63 & 2.15 & 2.03 & 2.05 & 2.43 & 5.42 & 2.02 & 2.04 & 2.04 & 2.08 & 2.59 \\
\hline $\operatorname{mean}\left(\sigma_{i}\right) \cdot \sqrt{V C_{i}}$ & 8.47 & 5.57 & 3.99 & 2.95 & 4.77 & 11.11 & 3.60 & 6.14 & 9.02 & 7.34 & 6.30 \\
\hline \multicolumn{12}{|c|}{ WETTZELL (Wettzell, Germany) } \\
\hline$V C_{i}$ & 4.45 & 3.45 & 1.50 & 1.39 & 3.49 & 2.32 & 2.56 & 2.26 & 18.90 & 10.13 & - \\
\hline $\operatorname{mean}\left(\sigma_{i}\right)$ & 2.87 & 1.73 & 1.47 & 1.88 & 1.78 & 4.64 & 1.77 & 1.44 & 1.64 & 1.53 & 2.07 \\
\hline $\operatorname{mean}\left(\sigma_{i}\right) \cdot \sqrt{V C_{i}}$ & 6.05 & 3.21 & 1.80 & 2.22 & 3.33 & 7.07 & 2.83 & 2.16 & 7.13 & 4.87 & 4.07 \\
\hline \multicolumn{12}{|c|}{ ZELENCHK (Zelenchukskaya, Russia) } \\
\hline$V C_{i}$ & 16.97 & 2.96 & 6.61 & 0.40 & 5.61 & 3.19 & 5.16 & 4.45 & 29.70 & 12.37 & - \\
\hline $\operatorname{mean}\left(\sigma_{i}\right)$ & 3.51 & 3.89 & 3.36 & 6.89 & 3.33 & 7.30 & 3.30 & 3.50 & 2.12 & 3.88 & 4.11 \\
\hline $\operatorname{mean}\left(\sigma_{i}\right) \cdot \sqrt{V C_{i}}$ & 14.46 & 6.69 & 8.64 & 4.36 & 7.89 & 13.04 & 7.50 & 7.38 & 11.55 & 13.65 & 9.52 \\
\hline
\end{tabular}


Table 7 Common noise parameters, $\bar{\alpha}^{2}$, and mean formal errors, $\bar{\sigma}$, of the combined ZTDs obtained by the unweighted, diagonal, variance components (VC), and OSI models; units of the formal errors are mm. The last row ('mean') gives the mean of the values in the above columns.

\begin{tabular}{|l|r||r|r|r|r|}
\hline Station & $\bar{\alpha}^{2}$ & unweighted $\bar{\sigma}$ & diagonal $\bar{\sigma}$ & VC $\bar{\sigma}$ & OSI $\bar{\sigma} \mid$ \\
\hline HARTRAO & 2.62 & 2.87 & 4.70 & 8.55 & 8.08 \\
KOKEE & 2.64 & 2.30 & 4.33 & 9.39 & 8.66 \\
MEDICINA & 2.24 & 2.36 & 4.91 & 12.37 & 10.21 \\
NYALES20 & 2.45 & 1.27 & 2.75 & 3.82 & 6.22 \\
ONSALA60 & 2.90 & 1.57 & 3.33 & 5.40 & 7.29 \\
SVETLOE & 2.41 & 2.00 & 4.08 & 8.32 & 8.41 \\
TIGOCONC & 2.71 & 3.64 & 5.43 & 7.93 & 8.20 \\
TSUKUB32 & 3.06 & 3.19 & 6.01 & 15.57 & 12.26 \\
WESTFORD & 2.50 & 2.05 & 4.18 & 8.86 & 8.86 \\
WETTZELL & 2.35 & 1.38 & 3.14 & 5.06 & 7.13 \\
ZELENCHK & 3.29 & 3.15 & 5.16 & 9.29 & 9.29 \\
\hline mean & 2.65 & 2.34 & 4.37 & 8.60 & 8.60 \\
\hline
\end{tabular}



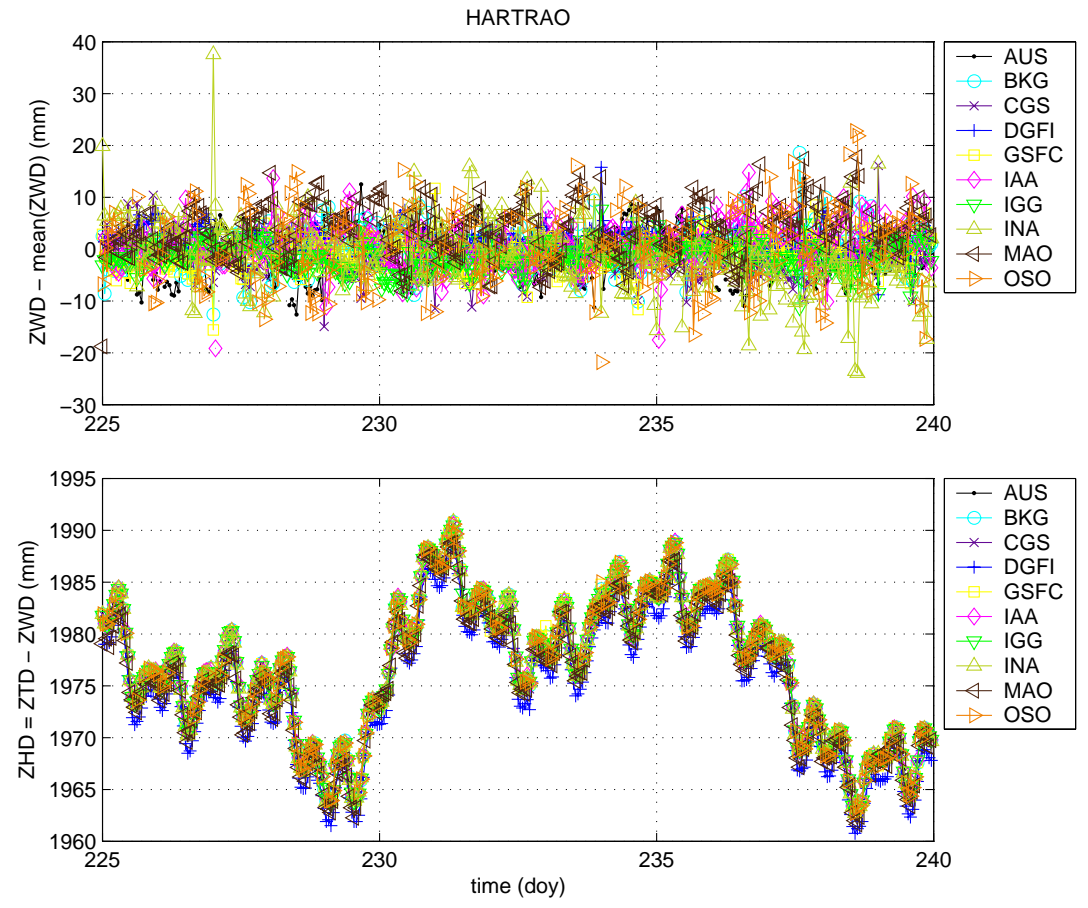

Fig. $1 Z W D$ differences $(t o p)$ to the unweighted mean of all ACs and derived $Z H D$ s (bottom) at station Hartebeesthoek, South Africa, provided by various IVS ACs. At this and other sites (not shown here) the $Z H D$ s show pronounced semidiurnal signals.
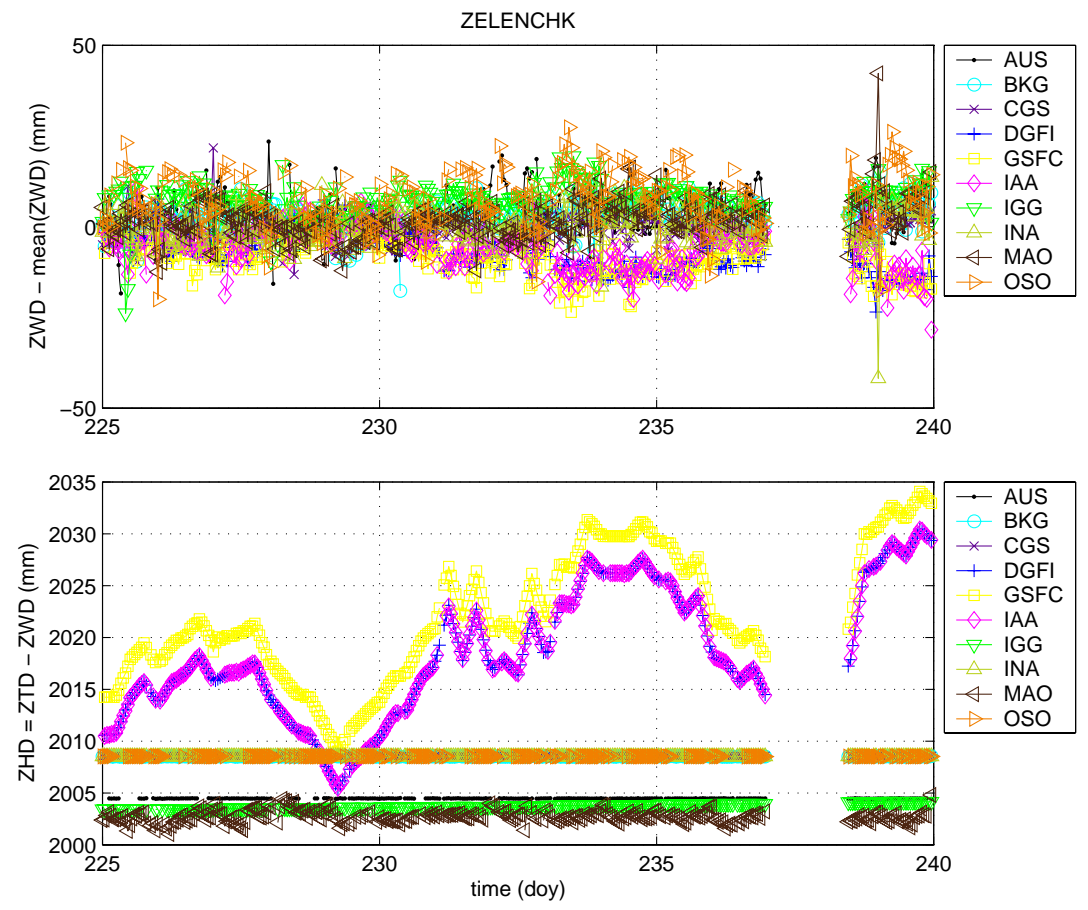

Fig. $2 Z W D$ differences ( $t o p)$ to the unweighted mean of all ACs and derived $Z H D$ s (bottom) at station Zelenchukskaya, Russia, provided by various IVS ACs. At this particular station no surface air pressure was recorded during CONT08. The differing $Z H D$ s and a significant part of the $Z W D$ differences arise from the various methods of substituting missing pressure values. 



Fig. 3 Effects of various apriori TRFs and handling of TRFs on the estimated troposphere parameters at Wettzell. "VTRF2008fix" denotes a solution based on the same TRF (VTRF2008) but without estimating session-wise adjustments to the station coordinates. With the other approaches, station coordinates are adjusted along with the troposphere parameters.
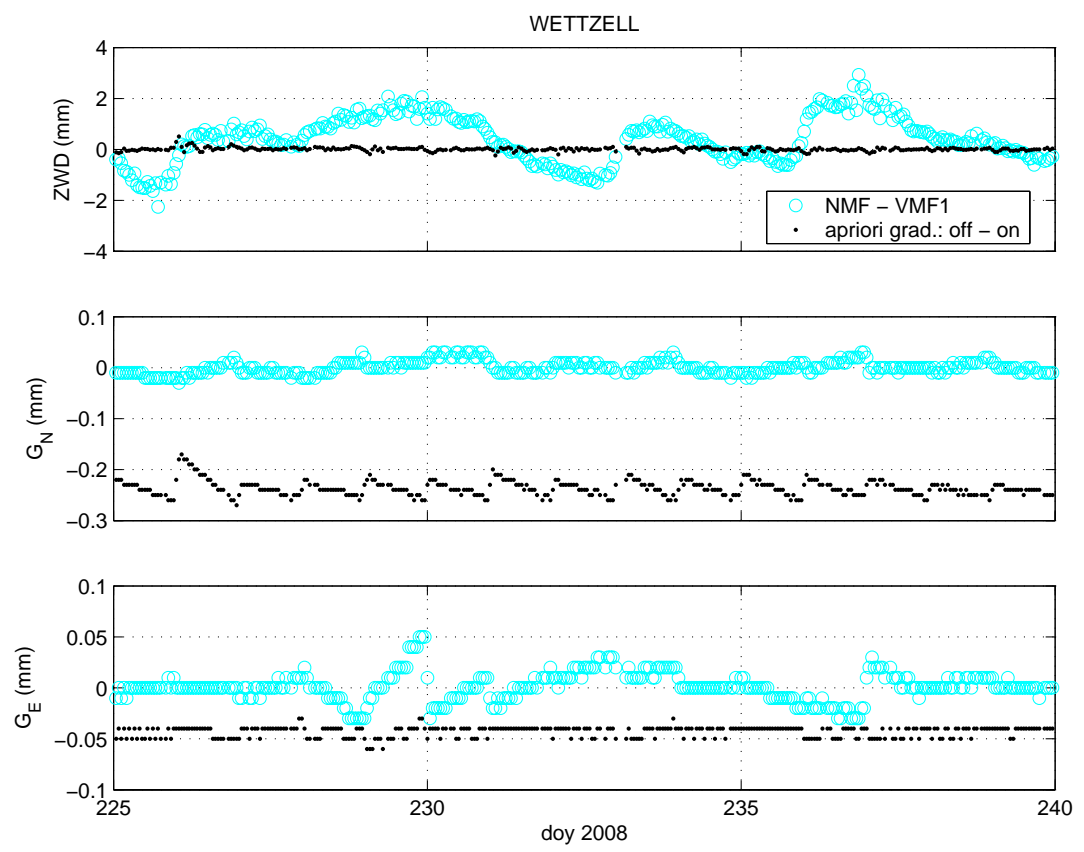

Fig. 4 Effects of mapping functions and apriori gradients on the estimated troposphere parameters at Wettzell. The adjusted gradients differ depending on the apriori gradients; this is due to the application of constraints. 



Fig. 5 Effects of parameterizations and constraints applied by several ACs on the estimated troposphere parameters at Wettzell. The parameterization and constraints of the other ACs using the Gauss-Markov-model are tested at DGFI with the OCCAM software against the solution of DGFI. BKG and INA apply identical parameterization and constraints for the troposphere parameters.
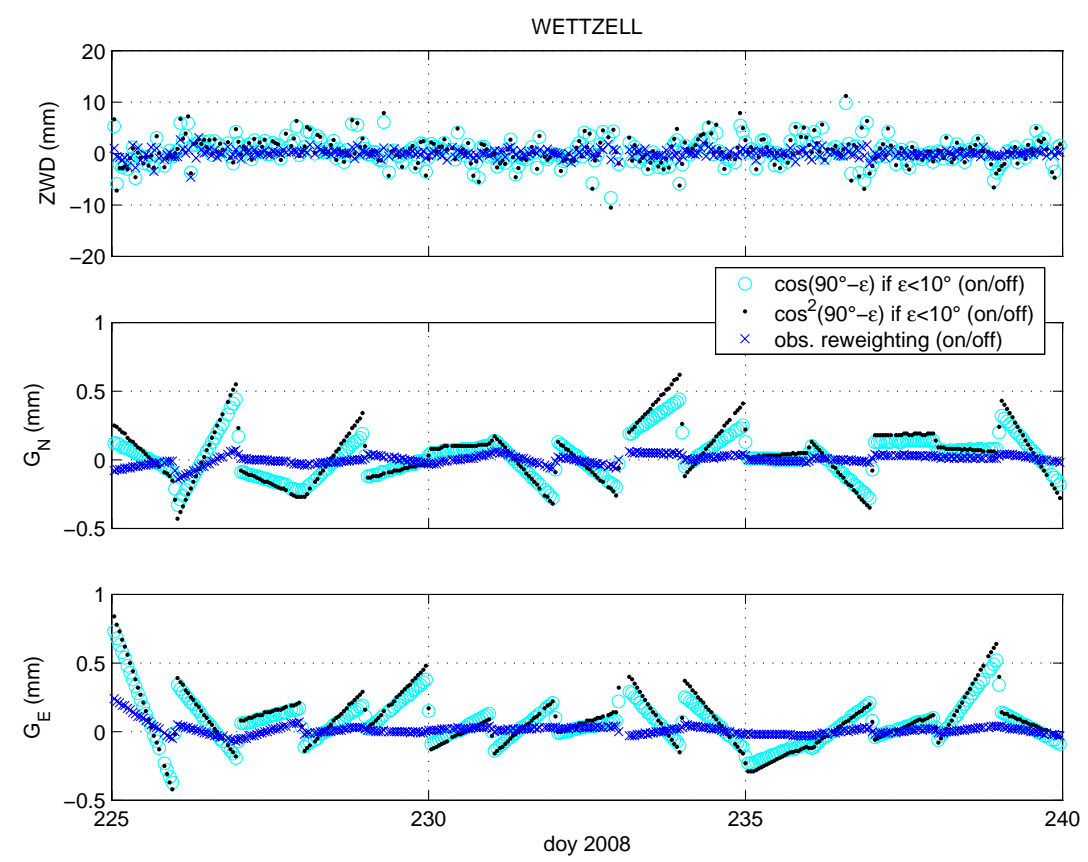

Fig. 6 Effects of data handling applied by several ACs on the estimated troposphere parameters at Wettzell. The various data handlings of other ACs are tested at DGFI with the OCCAM software and compared with the solution of DGFI. 



Fig. 7 Mean impact of the various stochastic models on the combined parameters (left) and relations of the mean formal errors of the combined $Z T D$ s with regard to the diagonal model (equ. 6) (right). 